\title{
Indicadores de uso sustentable del agua en Ciudad Juárez, Chihuahua
}

\author{
Luis Ernesto Cervera Gómez*
}

\begin{abstract}
Resumen. Este trabajo propone la generación de indicadores que permitan un avance en la evaluación del uso sustentable del agua en Ciudad Juárez, Chihuahua. Para la medición de sustentabilidad se propone un modelo sistemático que involucra a la sociedad, la infraestructura, las instituciones y el medio ambiente. Se logra avanzar en la generación y medición de indicadores que tienen que ver con la calidad de vida de la sociedad, el estado actual de la infraestructura de agua potable y la respuesta en materia de tratamiento. Estos indicadores son: 1. acceso al agua segura; 2 . acceso a instalaciones sanitarias adecuadas; 3 . conexiones domiciliarias; 4 . precio del agua; 5 . consumo del agua; y 6. aguas residuales tratadas. Los resultados indican que en Ciudad Juárez el sistema municipal de agua y saneamiento presenta buenos indicadores de uso sustentable del agua; esto sin considerar la tasa de extracción-recarga del acuífero y la calidad del agua potable. Quedan por trabajar los indicadores sobre las instituciones o el marco legal (gobernabilidad del agua), sobre participación ciudadana y sobre impacto al medio ambiente.

Palabras clave: ambiente, sustentabilidad, uso sustentable del agua, indicadores de sustentabilildad, Ciudad Juárez
\end{abstract}

\begin{abstract}
This paper seeks to develop indicators that allow us to progress in the evaluation of the sustainable use of water in Ciudad Juarez, Chihuahua. In order to measure sustainability, a systematic model was proposed involving society, institutions, infrastructure and the environment that would allow us to advance in the knowledge about interaction between society and its environment. Progress was achieved in the developing and measurement of some indicators, such as those related to quality of life and current state of water infrastructure and treatment. Using existing data, it was possible to develop and measure the following indicators for the sustainable use of water: 1 . secure access to water; 2 . access to adequate sanitation systems; 3 . domiciliary connections; 4 . water price; 5 . water consumption, and 6. waste water treatment. Results indicate that the municipal water system of Ciudad Juarez presents good indicators for sustainable use of water, however, they do not take into account the negative rate of rechargeextraction of the city's water supply system or of the quality of water for human consumption. The author considers that other indicators related to institutional and legal frame works, citizen participation, water quality, and environmental impact must be developed and estimated.

Key words: environment, sustainability, sustainable use of water, sustainability indicators, Ciudad Juárez
\end{abstract}

* Profesor-investigador en la Dirección General Regional Noroeste, El Colegio de la Frontera Norte, Ciudad Juárez, Chihuahua, México. Correo electrónico: lcervera@colef.mx 


\section{Introducción}

Ciudad Juárez se localiza en la región fronteriza conocida como Paso del Norte por colindar con la ciudad de El Paso, Texas. Las características de aridez de esta región, aunadas a un gran crecimiento poblacional cercano a los dos millones de habitantes, hacen que el agua sea un factor limitante para el desarrollo. Es por esto que conocer su manejo en términos de un marco de desarrollo sustentable es apremiante. En una fase inicial, el objetivo principal de este trabajo es la generación de indicadores de desarrollo sustentable del agua sólo para Ciudad Juárez, Chihuahua; esto es, los indicadores no revelarán, en este análisis, el contexto binacional. Para alcanzar el objetivo se propone trabajar bajo un modelo sistémico conformado por la sociedad y el medio ambiente, y así poder plantear un esquema que permita medir cómo se usa el agua en una sociedad y que además se pueda parametrizar de alguna manera su uso sustentable o su única alternativa: la insustentabilidad. Con la creación de indicadores sobre el uso y manejo del agua a escala municipal se puede ir haciendo de alguna manera más tangible el concepto de sustentabilidad dentro de un modelo sistemático que integre la coevolución de la sociedad con sus recursos hídricos. Creemos que la información generada en esta investigación puede ser utilizada como guía en las actuales políticas y en la toma de decisiones en materia de agua en los diferentes niveles de la sociedad y que los indicadores son perfectamente replicables para otras ciudades que cuenten con una Junta Municipal de Aguas con características similares a las de Ciudad Juárez.

Los indicadores son desarrollados a partir del uso de un modelo sistemático que incorpora los componentes principales de un sistema sustentable. Estos componentes son: la sociedad, las instituciones, la infraestructura y el ambiente. Partiendo del enfoque propuesto, así como de la información existente, se incorporan algunos indicadores propuestos en la agenda Hábitat 2004 y por la Secretaría de Desarrollo Social (Sedesol). Los indicadores desarrollados fueron estimados con información censal del INEGI, así como de la Junta Municipal de Agua y Saneamiento de Ciudad Juárez (JMAS), por lo que la metodología propuesta servirá de base para la réplica de estos indicadores para 
otros municipios fronterizos tal y como se diseñó en el proyecto "Observatorio Urbano de Ciudad Juárez" que actualmente se realiza en El Colegio de la Frontera Norte.

\section{Marco teórico-conceptual: el desarrollo sustentable}

El agua es una parte esencial de cualquier economía y sociedad, por lo tanto su manejo sustentable es una condición necesaria para una economía y una sociedad sustentables. Aunque el concepto de sustentabilidad puede tener varios significados para la sociedad y sus instituciones, Loucks y Gladwell (1999) señalan que la sustentabilidad es un concepto unificador, el cual enfatiza la necesidad de considerar el futuro a largo plazo tanto como el presente. La idea anterior incluye el futuro de la economía, el ambiente y los impactos sociales y ecológicos resultantes de las acciones y decisiones tomadas en el presente. Así tenemos que la sustentabilidad es un proceso integrador de aspectos tecnológicos, ecológicos y de las infraestructuras sociales y políticas de la sociedad.

Basados en conceptos generales de sustentabilidad, Gleick et al. (1996) definen al uso sustentable del agua como "el uso de agua que permite sostener a una sociedad para que perdure y se desarrolle en un futuro indefinido sin alterar la integridad del ciclo hidrológico y de los ecosistemas que dependan de él". Este concepto puede ser discutido como meramente filosófico (utópico) y difícil o imposible de alcanzar en la práctica. Sin embargo, la aproximación a un posible estatus de sustentabilidad requiere el establecimiento de criterios direccionados hacia los sistemas suministradores del recurso agua con el objetivo de ser considerados sustentables. Estos criterios pueden definirse respecto a: 1. diseño, manejo y operación de la infraestructura física; 2. calidad ambiental o salud de los ecosistemas; 3 . mantener el agua en estándares de calidad de acuerdo a su uso; 4 . contar con datos cualitativos y cuantitativos del agua; 5. desarrollar mecanismos institucionales para prevenir y resolver conflictos sobre el agua; 6 . que las partes involucradas participen en los procesos de diseño y decisión; y 7. las acciones humanas no deberán afectar las tasas de renovabilidad de las fuentes acuíferas y superficiales. Siguiendo las líneas generales puede iniciarse el diseño de indicadores de sustentabilidad. 
Sin duda, un componente muy fuerte en materia de sustentabilidad es el uso del agua con una explotación equilibrada de los componentes subterráneos y superficiales de agua dentro de una región hidrológica cuyos límites y potenciales pueden ser técnicamente conocidos. De acuerdo a la OECD (1998), en su documento titulado Towards Sustainable Development, el hecho de relacionar extracción de agua versus su tasa de renovabilidad es un punto central. Para determinar una extracción sustentable de agua renovable es importante conocer la tasa de extracción y el agua bombeada en un periodo dado en relación con el volumen total disponible al principio y al final del periodo establecido.

\section{¿Cómo medir el uso sustentable del agua?}

Bossel (1999) expresa que el desarrollo sustentable es una coevolución entre el ser humano y sus sistemas naturales, por lo que en materia del uso sustentable del agua podemos considerar que los elementos son la sociedad y el recurso hídrico. Con lo anterior puede establecerse un sistema que represente la interacción anteriormente descrita. Para el presente trabajo se explora un modelo sistemático que incorpora los componentes principales de un sistema sustentable. Éstos son: la sociedad, las instituciones, la infraestructura y el ambiente que sustenta al recurso agua (figura 1). Partiendo de este enfoque de sistema se propondrán indicadores de sustentabilidad.

Figura 1.

Componentes principales de un sistema sustentable

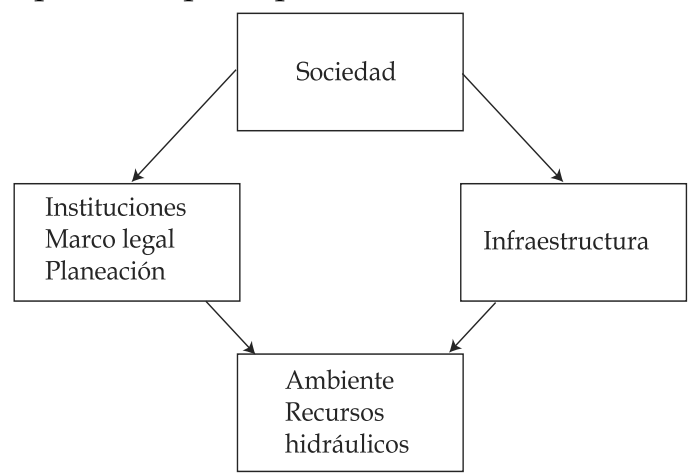


El sistema anterior podría contar con los siguientes subsistemas, considerados esenciales:

1. Sistema social: crecimiento poblacional, distribución del ingreso, estructura de clases, grupos sociales y organizaciones.

2. Gobierno: sistema legal, organismos operadores y planeación.

3. Infraestructura: red de agua potable, red de drenaje, baterías de pozos, distribución en pipas.

4. Sistema económico: producción y consumo, trabajo y empleo.

5. Recursos y ambiente: ambiente natural, atmósfera e hidrósfera, agotamiento de recursos naturales, reciclamiento, contaminación y degradación.

\section{Los indicadores y el sistema}

Una forma de medir el uso sustentable del agua puede ser a partir del desarrollo de indicadores que reflejen la interacción entre la sociedad y el recurso agua. Los indicadores proveen de información comprensiva acerca de los sistemas que dan forma al desarrollo sustentable, y son necesarios para guiar políticas y ayudar en la toma de decisiones en los diferentes niveles de la sociedad. De acuerdo con Bossel (1999), algunas de las características principales que deben tener los indicadores son las siguientes:

- Deben representar los problemas más importantes en la interacción de los sistemas y su ambiente.

- Deben ser claros y compactos, cubriendo los aspectos relevantes.

- El proceso de búsqueda del indicador debe ser participatorio para asegurar que incluya visiones y valores de la comunidad o región para la cual se desarrollan.

- Deben ser claramente definidos, reproducibles, no ambiguos, entendibles y prácticos.

- Deben ayudar a deducir la viabilidad y sustentabilidad de desarrollos actuales y permitir la comparación con proyectos alternativos. 
Estos indicadores deberán revelar las restricciones principales tanto físicas como humanas para el manejo sustentable del agua en la región. En las restricciones físicas los indicadores darán cuenta de la capacidad del sistema natural para brindar un recurso, tal como la tasa de renovabilidad del recurso agua en las zonas de captación.

Dentro del sistema o modelo sistemático arriba definido se pretende considerar un número compacto de indicadores cubriendo cada uno de los componentes y su interacción. Estos indicadores deberán, como ya se dijo, estar claramente definidos y ser reproducibles, entendibles y prácticos. Con las características anteriores, estos indicadores ayudarán a deducir la viabilidad y sustentabilidad del desarrollo actual a fin de compararlos con alternativas de desarrollo sustentable, así como a evaluar en forma simultánea los componentes ambientales y sociales del desarrollo sustentable. Una primera clasificación de indicadores hecha por Bossel (1999) los subdivide en indicadores de presión, estado y de respuesta. Éstos se describen a continuación:

Indicadores de presión. Permiten medir la presión sobre el ambiente causada por la actividad humana.

Indicadores de estado. Permiten medir la calidad del ambiente y la cantidad de recursos naturales, incluyendo efectos en la salud humana y de los ecosistemas causados por el deterioro del ambiente.

Indicadores de respuesta. Permiten medir los esfuerzos tomados por la sociedad para responder a los cambios ambientales.

\section{Ciudad Juárez y la región Paso del Norte}

Ciudad Juárez se localiza en la parte norte del estado de Chihuahua en una región conocida como Paso del Norte, llamada así desde antaño por ser un lugar de cruce del Río Bravo en la ruta México-Santa Fé, Nuevo México (figura 2). Esta región comprende además la ciudad de El Paso, Texas, y localidades del condado de Doña Ana, Nuevo México. Estas ciudades conforman una metrópoli de carácter binacional cuya población se aproxima a los dos millones de habitantes. De acuerdo al último censo, para el año 2000 Ciudad Juárez contaba con una 
Figura 2.

Localización de Ciudad Juárez, Chihuahua

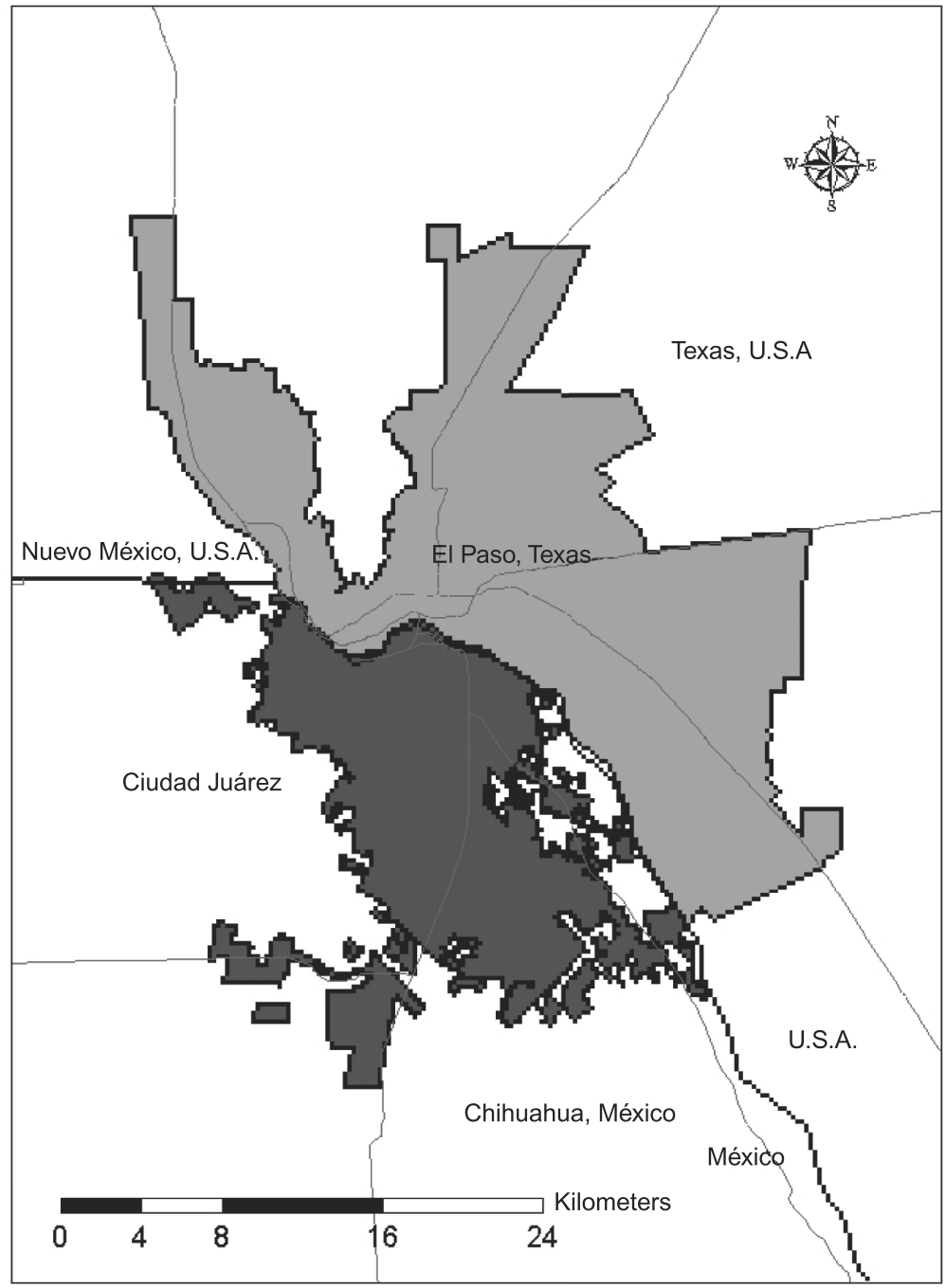


población de 1217818 habitantes (INEGI, 2000), representando un 61\% de la población de la región Paso del Norte. Para el mismo periodo censal, la ciudad de El Paso contaba con una población de 679622 a nivel condado (EPWU, 2006), representando el $34 \%$ de la región, perteneciendo el restante $5 \%$ a localidades del estado de Nuevo México. En esta región, tanto Ciudad Juárez como El Paso son las ciudades más importantes en términos demográficos y económicos dentro de su municipio o condado, respectivamente.

Como se ha explicado, desde la década de los cincuenta Ciudad Juárez se ha caracterizado por experimentar altas tasas de crecimiento poblacional a ritmos que la colocaron como una de las ciudades de mayor crecimiento a nivel poblacional. Así, de 1950 a 1990 la ciudad experimentó un crecimiento medio anual del 5.8\% (cuadro 1). Se da por entendido el hecho de que la mayor parte de este dinamismo poblacional se debió a la atracción que ha ejercido la ciudad a los flujos migratorios como resultado de su localización en cuanto puerto fronterizo y al acelerado proceso de industrialización vía maquiladora. Este crecimiento poblacional tuvo un impacto directo en el proceso de expansión urbana de Ciudad Juárez. Así, desde la década de los cincuenta la ciudad se embarcó en una fase de expansión física (Fuentes, 2001). En 1950, la mancha urbana ocupó una superficie de 800 hectáreas y para 1990 alcanzó 14 049, es decir, se incrementó en 13249 hectáreas. Asimismo, la densidad de población disminuyó en el mismo periodo de 164 a 57 habitantes por hectárea. Es decir, durante el periodo la ciudad experimentó un patrón de crecimiento expansivo. Estos ritmos de crecimiento poblacional y expansión territorial de la ciudad son expresados en el cuadro 1 (Fuentes y Cervera, 2004). Lo anterior está directamente relacionado con un incremento en la presión sobre el recurso agua capaz de soportar los cambios poblacionales.

\section{Medio físico}

\section{Clima}

De acuerdo con la clasificación climática de Köeppen, la región de Ciudad Juárez está clasificada como clima seco desértico con veranos 
Cuadro 1. Crecimiento poblacional y urbano de Ciudad Juárez, 1856-2000

\begin{tabular}{rrrrc}
\hline Año & Población & $\begin{array}{c}\text { Tasa de } \\
\text { crecimiento de } \\
\text { la población (\%) }\end{array}$ & $\begin{array}{c}\text { Área urbana } \\
\text { (hectáreas) }\end{array}$ & $\begin{array}{c}\text { Densidad de } \\
\text { población } \\
\text { Población/hectárea }\end{array}$ \\
\hline 1856 & 4342 & & 9 & 482 \\
1894 & 7582 & 1.4 & 60 & 126 \\
1900 & 8218 & 3.5 & 61 & 134 \\
1910 & 10621 & 2.5 & 119 & 89 \\
1921 & 19457 & 5.5 & $\mathrm{ND}$ & $\mathrm{ND}$ \\
1930 & 39669 & 7.9 & 471 & 84 \\
1940 & 48881 & 2.0 & 563 & 87 \\
1950 & 131308 & 9.1 & 800 & 164 \\
1960 & 276995 & 7.2 & 1894 & 146 \\
1970 & 424135 & 5.2 & 5608 & 75 \\
1980 & 567365 & 4.4 & 9395 & 60 \\
1990 & 798499 & 3.4 & 14049 & 57 \\
2000 & 1217818 & 4.2 & 21572 & 56 \\
\hline
\end{tabular}

Fuente: César Fuentes (2001: 95-118).

cálidos e inviernos frescos a fríos (Bwk) o muy seco templado. Cuenta con una temperatura media mensual de 18 grados centígrados estimada para el periodo 1957-2001 (cuadro 2). Durante el mismo periodo, esta región presenta un promedio de precipitación anual de aproximadamente 262 milímetros (mm). En 1964, considerado el año más seco, se estimó una precipitación de $119.6 \mathrm{~mm}$. Contrariamente, durante el año más lluvioso (1989) se registró una precipitación total anual de $536.3 \mathrm{~mm}$ (cuadro 3).

\section{Aguas superficiales y subterráneas}

El Río Grande y los acuíferos transfronterizos en la región de Las Cruces, Nuevo México, El Paso, Texas, y Ciudad Juárez, México, constituyen el único recurso hídrico para una población aproximada de dos millones de personas y una superficie irrigada de aproximadamente 
Cuadro 2. Temperaturas medias mensuales para Ciudad Juárez, Chihuahua

\begin{tabular}{lrrrr}
\hline Mes & $1957-2001$ & 2001 & 2001 (Max) & 2001 (Min) \\
\hline Ene & 7.2 & 7.8 & 23.0 & -6.0 \\
Feb & 10.3 & 10.9 & 27.0 & -2.0 \\
Mar & 13.4 & 15.3 & 33.0 & 0.0 \\
Abr & 18.1 & 21.0 & 36.0 & 5.0 \\
May & 22.7 & 26.1 & 40.0 & 11.0 \\
Jun & 27.2 & 29.6 & 41.0 & 12.0 \\
Jul & 28.3 & 30.1 & 41.0 & 20.0 \\
Ago & 27.1 & 29.2 & 39.0 & 18.0 \\
Sep & 24.2 & 25.3 & 38.0 & 12.0 \\
Oct & 18.4 & 21.0 & 32.0 & 8.0 \\
Nov & 11.9 & 15.4 & 34.0 & -4.0 \\
Dic & 7.5 & 8.7 & 25.0 & -6.0 \\
\hline
\end{tabular}

Fuente: CNA, Registro mensual de temperatura media, INEGI, Anuario estadístico, 2002.

Cuadro 3. Precipitación media mensual en Ciudad Juárez, Chihuahua

\begin{tabular}{lrrrr}
\hline & $1957-2001$ & 2001 & $\begin{array}{c}\text { Año más seco } \\
(1964)\end{array}$ & $\begin{array}{c}\text { Año más lluvioso } \\
(1989)\end{array}$ \\
\hline Ene & 13.4 & 2.5 & 2.5 & 4.4 \\
Feb & 10.4 & 0.0 & 7.5 & 25.3 \\
Mar & 7.9 & 11.0 & 10.5 & 14.7 \\
Abr & 5.4 & 0.0 & 1.0 & 0.0 \\
May & 9.6 & 0.0 & 0.1 & 15.9 \\
Jun & 17.8 & 25.0 & 1.0 & 0.6 \\
Jul & 54.9 & 23.0 & 27.0 & 360.2 \\
Ago & 49.8 & 35.7 & 34.0 & 22.8 \\
Sep & 40.2 & 27.0 & 15.0 & 10.7 \\
Oct & 21.0 & 0.0 & 6.0 & 0.1 \\
Nov & 12.3 & 23.0 & 0.0 & 5.9 \\
Dic & 18.9 & 3.0 & 15.0 & \\
Anual & 261.6 & & & \\
\hline
\end{tabular}

Fuente: CNA, Registro mensual de precipitación media, INEGI, Anuario estadístico, 2002. 
170 mil acres -aproximadamente 78 mil hectáreas (Hibbs et al., 2002). La figura 3 muestra la localización de los principales acuíferos de donde se extrae el agua en esta región, además de resaltar la configuración de las manchas urbanas y el Valle de Juárez.

Las ciudades de El Paso y Juárez están asentadas sobre el acuífero conocido como Bolsón del Hueco. Sin embargo, el abastecimiento de agua potable se establece en diferentes proporciones. Así, de este acuífero Ciudad Juárez obtiene el 100\% para su consumo de agua potable y municipal, y a corto plazo se comenzará a transferir agua del Bolsón de la Mesilla. En el caso de El Paso, esta ciudad extrae del Bolsón del Hueco aproximadamente 47\%; 20\% proviene del Bolsón de la Mesilla y 33\% de aguas del Río Bravo. Para el uso agrícola, el agua proviene en su totalidad de los escurrimientos del Río Bravo (Rincón et al., 2005).

Las aguas de Bolsón del Hueco han mantenido el crecimiento de esta región del Paso del Norte. Sin embargo, las tasas de extracción exceden en gran medida la tasa de recarga marcando drásticamente una condición de uso no sustentable del recurso hídrico. Hibbs et al. (2002) reportan que la tasa de extracción es de 15 a 20 veces mayor

Figura 3.

Extracción mensual de agua en Ciudad Juárez, Chihuahua

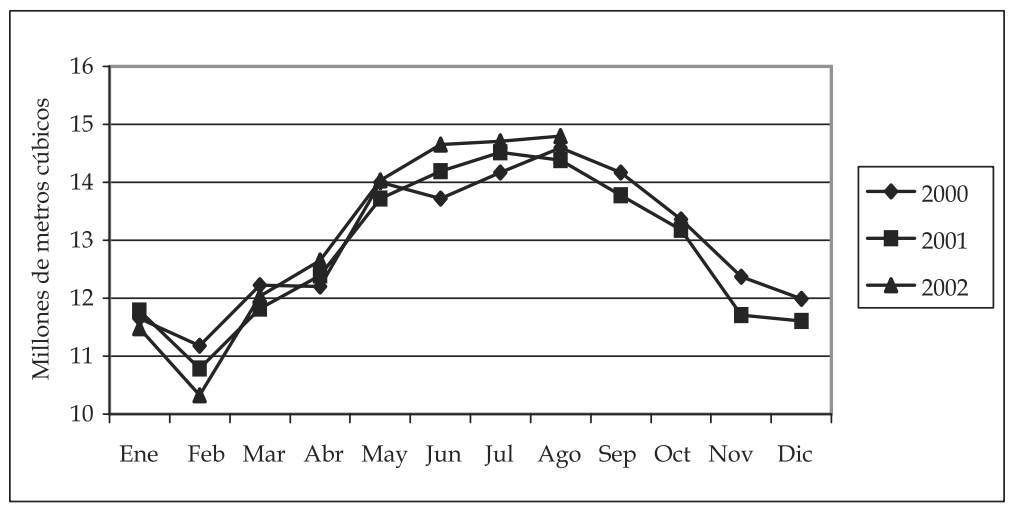

Fuente: JMAS (2002). 
que la tasa de recarga. Consecuentemente, el nivel freático ha descendido drásticamente favoreciendo un proceso de salinización del agua. Por ejemplo, se ha reportado que los niveles de cloruro rebasan ahora el máximo nivel recomendado para consumo humano, que es de 250 miligramos por litro.

A medida que el agua subterránea se mueve desde sus áreas de recarga hacia las áreas de descarga, su calidad evoluciona geoquímicamente. Así, el agua en las áreas de recarga se caracteriza por contener bajas concentraciones de iones disueltos, dominando los iones como calcio, magnesio y bicarbonato. A medida que nos acercamos a las zonas de descarga existe un incremento en la concentración de sólidos totales disueltos, resultando en un incremento de iones de sodio aunado con una reducción de las concentraciones de calcio y magnesio. Las concentraciones de sulfatos y cloruros también se incrementan debido a la disolución de minerales de evaporita, tales como el yeso (Hibbs et al., 2002). Es por esta razón que la ciudad de El Paso ha decidido abandonar algunos de sus pozos. Algunos expertos predicen que este acuífero será agotado para el año 2030 (Bixby, 1999). Este proceso de salinización experimentado en la ciudad de El Paso pronto podría repetirse para el acuífero de Ciudad Juárez, con el consecuente abandono de pozos y el planteamiento de otras alternativas.

\section{Ciudad Juárez: aguas superficiales y subterráneas}

Ciudad Juárez dispone de un suministro anual de 60 mil acres-pies (aproximadamente $74 \mathrm{~mm}^{3}$ ) de las aguas del Río Bravo, de acuerdo al tratado internacional firmado por México y Estados Unidos en 1906. Esta agua es entregada en la ciudad, canalizada a través de acequias $\mathrm{y}$, posteriormente, mezclada con aguas residuales y usadas para riego agrícola en el Valle de Juárez. En conclusión, las aguas superficiales del Río Bravo, que de acuerdo al tratado internacional ingresan a Ciudad Juárez, no tienen un uso urbano, utilizándose en un 100\% para irrigación en el Valle de Juárez. Sin embargo, estas aguas podrían considerarse en un futuro para su potabilización e incorporación al sistema municipal de agua potable y alcantarillado. 
El Río Grande es la única fuente renovable de agua para la región de Ciudad Juárez-El Paso. Como ya se mencionó, Ciudad Juárez depende totalmente de Bolsón del Hueco. Para la extracción del agua demandada por la ciudad se cuenta con un total de 180 pozos perforados. De éstos, 160 están equipados y sólo 145 están en operación. De acuerdo con JMAS (2002), se tiene una gasto promedio por pozo de aproximadamente 41.59 litros por segundo (lps).

En 1990 se extraía un volumen anual de 119.8 millones de metros cúbicos $\left(\mathrm{mm}^{3}\right)$. El bombeo ha aumentado a una tasa anual del $2.5 \%$ en promedio. Así, para el año 2000 se reporta un bombeo de $153 \mathrm{~mm}^{3}$ y para el año 2005 se reporta una extracción por un total de $147.3 \mathrm{~mm}^{3}$. Lo anterior revela una aparente disminución en la extracción de agua del acuífero, y según reportes de la JMAs esto tiene que ver con la disminución del consumo per cápita. En Ciudad Juárez la extracción se realiza mediante una batería compuesta de 130 a 145 pozos, con un bombeo diario de 5600 litros por segundo por día -lt/seg/ día- (JMAS, 2005). La cifra anterior equivale a una extracción anual aproximada a los $176 \mathrm{~mm}^{3}$. Este bombeo provee una capacidad de servicio de 330 litros por habitante por día. Sin embargo, la dotación per cápita promedio es un poco menor y el organismo operador reporta que este indicador se ha estado reduciendo significativamente.

Es importante destacar la alta variabilidad del volumen de extracción mensual debido a las condiciones climáticas. Por ejemplo, en el 2002 se sostuvo un volumen de extracción mensual promedio de 12.82 $\mathrm{mm}^{3}$. Durante ese año se mantuvo la extracción mínima mensual durante el mes de febrero $\left(10.786 \mathrm{~mm}^{3}\right)$, y una extracción máxima mensual de $14.51 \mathrm{~mm}^{3}$ durante el mes de septiembre (figura 4).

\section{Recarga de aguas subterráneas vs. extracción en Ciudad Juárez}

Se estima que el acuífero de Bolsón del Hueco, tan sólo en el área de extracción de agua por la JMAS en una superficie considerada de 23 260.89 hectáreas (extensión aproximada a la mancha urbana de Ciudad Juárez), tiene una recarga anual de $35 \mathrm{~mm}^{3}$ anuales. Esto significa que al menos en Ciudad Juárez la extracción supera en aproximadamente cinco veces la recarga estimada por concepto de precipitación 
(JMAS, 2005). Sin embargo, el acuífero también recibe recargas subterráneas en un gradiente hidráulico norte-sur proveniente del lado estadounidense, mismas que no están debidamente cuantificadas. Se requiere realizar un balance hidrológico para Bolsón del Hueco considerando la totalidad de su área asentada en ambos países.

\section{El Paso: aguas superficiales y subterráneas}

En la ciudad de El Paso el consumo per cápita ha sido, y es en la actualidad, significativamente mayor que en Ciudad Juárez. El organismo operador llamado El Paso Water Utilities (EPWU) reporta para 1971 un consumo de 220 galones por persona por día (equivalente a 850 litros por persona por día). Sin embargo, las políticas de ahorro han disminuido el nivel de consumo a niveles menores de los 140 galones por persona (543 litros por persona por día) (EPWU, 2006). No obstante, su tamaño poblacional y la existencia de otras fuentes (La Mesilla y Río Grande) hacen que la ciudad de El Paso realice un volumen de extracción (de Bolsón del Hueco) menor que en Ciudad Juárez. ELPWU (2006) reporta una extracción de $44.4 \mathrm{~mm}^{3}$ en 1967, con un pico de extracción de $97.03 \mathrm{~mm}^{3}$ en 1989 y finalizando en el 2002 con una extracción equivalente a los $48.27 \mathrm{~mm}^{3}$ (figura 4). La última cantidad denota que la extracción actual de ambas ciudades es en proporción 3 a 1 para Juárez y El Paso, respectivamente.

\section{Recarga de aguas subterráneas vs. extracción en El Paso, Texas}

En este indicador de recarga se tienen estudios más completos en el área estadounidense. Se estima que en la porción de Bolsón del Hueco utilizada por la ciudad de El Paso se tiene un almacenamiento total de 9 millones de acres-pies de agua "dulce", lo que equivale a un volumen de almacenamiento total de $11.097 \mathrm{~mm}^{3}$; esto sin considerar el volumen de aguas saladas también existentes en el acuífero. De esta porción de agua no salobre, un modelo desarrollado por Heywood y Yager (2003) indica que la cantidad anual de agua removida de esta fuente varía en un rango entre 18 mil y 33 mil acres-pies por año (de 
Figura 4.

Regimen anual de extracción de agua del acuífero:

Bolsón del Hueco (1926-2002)

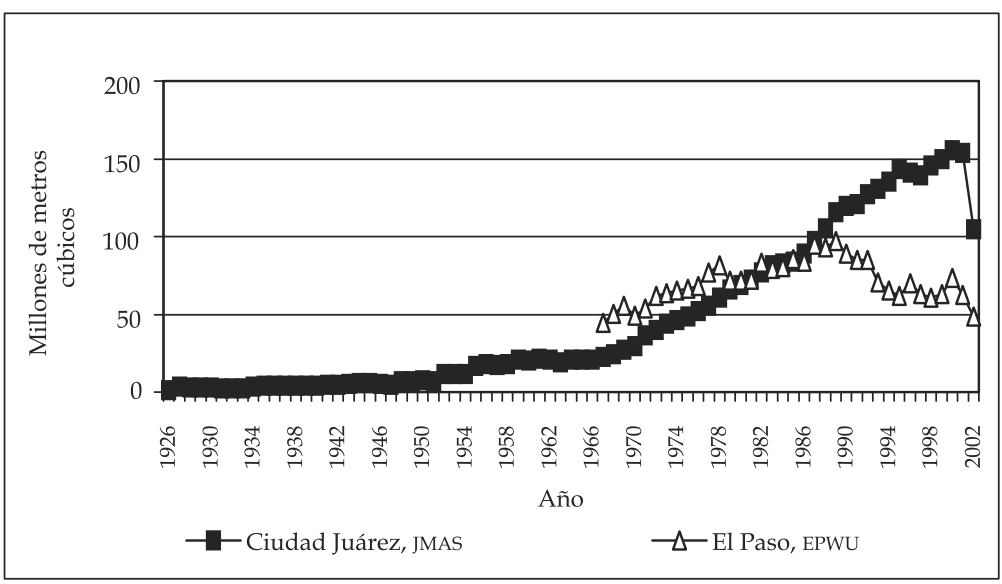

Fuente: JMAs, 2002.

22.194 a $40.689 \mathrm{~mm}^{3}$ ). En cuanto a la recarga, el modelo de Heywood y Yager estima que el agua superficial asociada al Río Grande recarga las aguas subterráneas del sistema acuífero a una tasa de 50 mil acrespies por año, cifra que equivale a $61.65 \mathrm{~mm}^{3}$ de recarga anual. Considerando una extracción anual tan sólo de Bolsón del Hueco, se tiene que la cantidad de agua extraída no es mayor a la de recarga reportada en el modelo arriba descrito. El modelo también sugiere que podría haber un suministro seguro de esta fuente para los próximos 70 años. Sin embargo, estas reservas pueden ser fuertemente impactadas por futuros crecimientos poblacionales tanto en El Paso como en Ciudad Juárez, donde actualmente Bolsón del Hueco es su única fuente para uso municipal.

\section{Tarifas de agua}

El sistema municipal de agua potable de Ciudad Juárez (JMAS) tiene cuatro tipos de usuarios principales; éstos están clasificados en do- 
mésticos, comercial, industrial y público (cuadro 4). Además, los usuarios domésticos han sido clasificados en cuatro categorías de acuerdo a su nivel socioeconómico. Así, del más bajo al más alto tenemos las clases 1 . baja; 2. mediana a baja; 3. mediana; y 4. mediana-alta. El criterio utilizado para esta clasificación incluye el tipo de vivienda y su localización física dentro del área urbana. La porción de agua utilizada por los cuatro sectores principales se estimó como sigue: doméstico $(81.7 \%)$; comercial (7.7\%); industrial (7.9\%); y público (3.56\%) (JMAS, 2000).

Las tarifas para el agua se han establecido por rangos correspondiendo a un sistema de cobro diferenciado donde el que gasta más paga más, además de considerar diferentes costos de acuerdo a su uso, asignándole un costo menor al de uso doméstico que al de los usos comerciales e industriales. Así, la JMAs estableció los rangos de uso en metros cúbicos, así como el costo (cuadros 5 y 6).

Ciudad Juárez ocupa un lugar importante en materia de recaudación por concepto de agua en el contexto estatal y nacional. A escala nacional, el promedio de recaudación por habitante reportado por la Comisión Nacional del Agua (CNA) en 1999 era de 1.45 pesos por metro cúbico por habitante, mientras que el promedio para el estado de

\section{Cuadro 4.}

Promedio de agua servida por tipo de usuario

\begin{tabular}{lll}
\hline Usuario & $\begin{array}{l}\text { Agua servida } \\
\text { Litros por habitante } \\
\text { por día }\end{array}$ & $\begin{array}{l}\text { Consumo mensual } \\
\left(\mathrm{m}^{3} / \text { toma }\right)\end{array}$ \\
\hline
\end{tabular}

Doméstico 31.38

Bajo (34.6\%)

270

Mediano-bajo (41.69 \%) 339

Mediano $(15.24 \%) \quad 386$

Mediano-alto $(8.47 \%) \quad 521$

Comercial

88.06

Industrial

802.06

Público

402.30

Pipas

7.79

Fuente: Servicios de Ingeniería e Informática (2000). 


\section{Cuadro 5.}

Tarifas de agua para uso doméstico por cuotas fijas y medidas

\begin{tabular}{lrrrrrr}
\hline \multirow{2}{*}{$\begin{array}{l}\text { Rango } \\
\left(\mathrm{m}^{3}\right)\end{array}$} & \multicolumn{3}{c}{ Tarifas con medición } & \multicolumn{3}{c}{ Tarifas fijas } \\
\hline $0-20$ & 1.77 & 0.70 & 2.47 & 2.22 & 0.88 & 3.10 \\
$21-30$ & 2.50 & 0.92 & 3.42 & 3.12 & 1.15 & 4.27 \\
$31-40$ & 3.22 & 0.92 & 4.14 & 4.03 & 1.15 & 5.18 \\
$41-50$ & 4.03 & 0.92 & 4.95 & 5.04 & 1.15 & 6.19 \\
$51-75$ & 5.12 & 0.98 & 6.10 & 6.41 & 1.22 & 7.63 \\
$76-100$ & 6.39 & 0.98 & 7.37 & 8.00 & 1.22 & 9.23 \\
$101-150$ & 8.43 & 1.17 & 9.60 & 10.55 & 1.48 & 12.02 \\
$>150$ & 10.35 & 1.17 & 11.53 & 12.97 & 1.48 & 14.45 \\
\hline
\end{tabular}

Fuente: JMAS, 2003.

Cuadro 6.

Tarifas de agua para usos comercial e industrial

\begin{tabular}{lrrr}
\hline $\begin{array}{l}\text { Rango } \\
\left(\mathrm{m}^{3}\right)\end{array}$ & Agua (A) & Drenaje (D) & \\
\hline $0-33$ & 2.67 & 0.75 & A+ D \\
$34-50$ & 6.54 & 0.92 & 4.42 \\
$51-75$ & 4.59 & 0.92 & 5.51 \\
$76-100$ & 5.07 & 0.98 & 6.05 \\
$101-150$ & 6.08 & 1.07 & 7.15 \\
$151-200$ & 7.05 & 1.22 & 8.27 \\
$201-300$ & 7.49 & 1.28 & 8.76 \\
$301-400$ & 7.73 & 1.36 & 9.09 \\
$401-500$ & 8.31 & 1.46 & 9.78 \\
$501-600$ & 8.86 & 1.52 & 10.37 \\
$601-1000$ & 13.66 & 2.28 & 15.94 \\
$1001-2000$ & 16.59 & 2.63 & 19.23 \\
$>2001$ & 19.48 & 2.76 & 22.24 \\
\hline
\end{tabular}

Fuente: JMAS, 2003. 


\section{Cuadro 7.}

Comparación de la tarifa de agua en Chihuahua vs. otras ciudades

\begin{tabular}{lrrrrrr}
\hline $\begin{array}{l}\text { Consumo } \\
\left(\mathrm{m}^{3}\right)\end{array}$ & Chihuahua & Hermosillo & $\begin{array}{l}\text { Cd. } \\
\text { Juárez }\end{array}$ & Monterrey & Tijuana & Torreón \\
\hline 10 & 47 & 25 & 47 & 50 & 52 & 34 \\
20 & 74 & 50 & 47 & 100 & 120 & 77 \\
30 & 116 & 78 & 85 & 171 & 356 & 122 \\
40 & 226 & 116 & 149 & 263 & 610 & 231 \\
50 & 294 & 163 & 187 & 373 & 870 & 235 \\
60 & 368 & 326 & 304 & 495 & 1220 & 282 \\
70 & 458 & 489 & 355 & 625 & 1434 & 384 \\
100 & 736 & 1010 & 613 & 1049 & 2049 & 549 \\
110 & 836 & 1198 & 879 & 1203 & 2254 & 869 \\
\hline
\end{tabular}

Fuente: JMAS, 2001.

Chihuahua se estimó en 1.74 pesos por metro cúbico. Para 1999 Ciudad Juárez recaudaba en promedio 2.25 pesos por metro cúbico por habitante (1.5 veces la media nacional). En el mismo reporte de la CNA tenemos que de un total de 942 localidades, Juárez ocupa el lugar número 20 a nivel nacional, sólo superado por algunas ciudades mexiquenses, las principales ciudades de Baja California y la ciudad de Morelia. Así, nuestra ciudad es por mucho la mayor recaudadora por metro cúbico de agua por persona en el estado. A la fecha los datos han cambiado de manera significativa y seguramente la recaudación per cápita es aún mayor.

En el caso de Ciudad Juárez la tarifa promedio es a razón de cuatro pesos el metro cúbico.

Con respecto a las tarifas domésticas, el cuadro 7 muestra el precio del agua en promedio para el estado de Chihuahua, comparado con otras ciudades principales en el norte de México.

\section{Medición del agua}

Para el año 2000 la ciudad contaba con un total de 265299 cuentas registradas. De éstas, tan sólo 59\% de los usuarios contaba con 
medidores y de éstos sólo $66 \%$ funcionaba en forma debida. Para el año 2002 se tienen contabilizadas 249102 cuentas contratadas (JMAS, 2002). Para el 2005, la JMAS reporta un total de 350 mil tomas domiciliarias y reconoce que la ciudad tiene un rezago del $27 \%$ en la cobertura de servicio medido. Se anuncia la instalación de $40 \mathrm{mil}$ medidores en las zonas faltantes a fin de incrementar el cobro por servicio medido.

\section{Tratamiento de aguas residuales}

Actualmente Ciudad Juárez cuenta con dos plantas principales de tratamiento de aguas residuales, éstas son la Planta de Tratamiento Norte y la Planta de Tratamiento Sur, con una capacidad de 2.5 y $1 \mathrm{~m}^{3} /$ seg. respectivamente (figura 5). Las plantas de tratamiento norte y sur cuentan con un tipo de tratamiento conocido como Primario Avanzado (TPA). Se considera que su efluente tiene una calidad que puede ser reutilizado en riego agrícola (JMAS, 1997). Este proceso básicamente consiste en sedimentación, desbaste de gruesos y tratamiento por clarifloculación y cloración.

Ambas plantas están ubicadas en Distrito de Riego 009 del Valle de Juárez. Esto representa una capacidad actual conjunta de $3.5 \mathrm{~m}^{3} / \mathrm{seg}$. Cabe destacar que la planta sur fue diseñada para ampliaciones que pudieran tratar hasta $3.5 \mathrm{~m}^{3} / \mathrm{seg}$. En el supuesto de un tratamiento constante, estas plantas tendrían la capacidad de dar tratamiento a un volumen anual de $110.376 \mathrm{~mm}^{3}$, representando este volumen tratado un $70 \%$ del volumen total alumbrado en el abastecimiento de la ciudad. Además se tienen proyectadas tres plantas de tratamiento, éstas son: Anapra (JMAS, 2005), con una capacidad de tratamiento de 180 litros/seg.; Chaparral, con una capacidad de 90 litros/seg.; y Laguna de Patos, con 800 litros/seg (JMAS, 2005). Así, con estas nuevas plantas se tendría una capacidad de tratamiento de aproximadamente 1.07 $\mathrm{m}^{3} / \mathrm{seg}$, así como una capacidad total estimada de $141.912 \mathrm{~mm}^{3}$ anuales, representando un tratamiento equivalente al $89 \%$ del volumen actual extraído o alumbrado.

Una vez tratadas, las aguas residuales son utilizadas para riego en el Valle de Juárez, mismo que tiene una superficie de 15450 hectáreas 
Figura 5.

Localización de zonas acuíferas, Valle de Juárez y Plantas de Tratamiento de Aguas Residuales en Ciudad Juárez

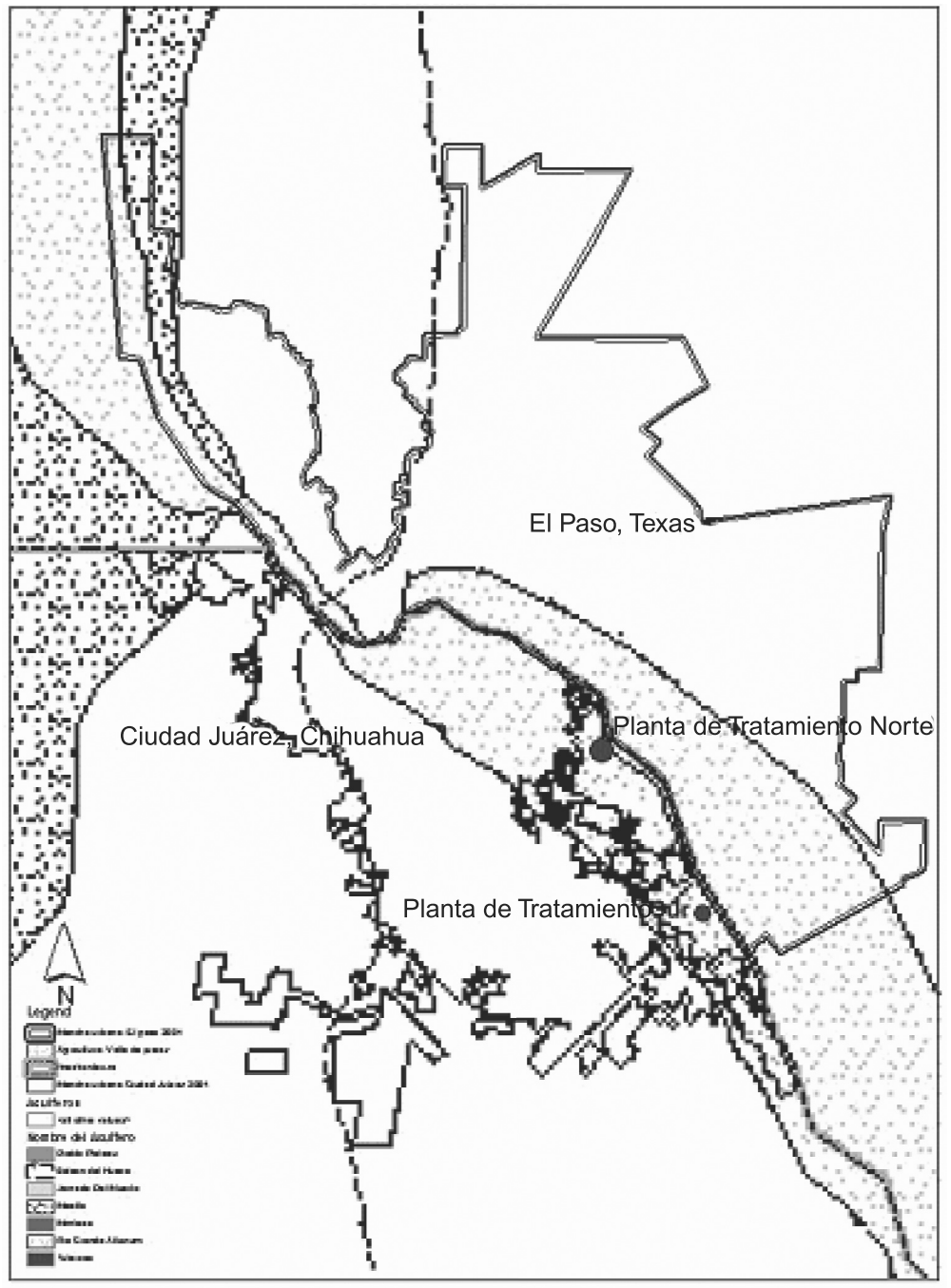


y que antes se irrigaba con aguas residuales crudas mezclada con agua bombeada del subsuelo.

\section{Estimación de indicadores del uso sustentable del agua}

Siguiendo las líneas generales puede iniciarse el diseño de indicadores de sustentabilidad. Para el caso específico de Ciudad Juárez, los indicadores fueron seleccionados estratégicamente en función de los datos disponibles, así como tratando de cubrir los aspectos ambientales, sociales, técnicos y administrativos de un sistema municipal de agua potable y en términos de un uso sustentable. Ahora bien, más allá de cumplir con aquellos indicadores desarrollados en la agenda Hábitat y por Sedesol, en materia del uso sustentable del agua pensamos que deben proponerse los indicadores que se describen a continuación:

Ambientales e infraestructura

1. Volumen mensual total alumbrado.

2. Abatimiento del acuífero.

3. Eficiencia de la red de distribución.

4. Relación entre suministro/drenaje.

5. Pérdidas por fugas de la red.

6. Aguas tratadas.

7. Calidad del efluente.

8. Reuso de aguas tratadas.

9. Producción promedio de lodos.

10. Disposición de lodos.

\section{Administrativos}

11. Precio del agua.

12. Relación entre volumen facturado/ volumen alumbrado.

13. Cobertura de medición volumétrica.

14. Capacidad de cobranza. 


\section{Sociales}

15. Relación ingreso-costo del agua.

16. Seguridad de suministro (consumo de agua).

17. Calidad del agua.

18. Acceso a instalaciones sanitarias adecuadas.

19. Conexiones domiciliarias.

\section{Indicadores desarrollados}

En esta sección se presenta, a manera de avance, una serie de indicadores que pueden ser perfectamente medibles para Ciudad Juárez. Para su presentación se elaboró una ficha que presenta su descripción, justificación, variables, algoritmo y cálculo. Estos indicadores forman parte del proyecto "Observatorio Urbano de Ciudad Juárez" en su apartado de medio ambiente.

\section{Acceso a agua segura (AAS)}

\section{Descripción}

Consiste en estimar la proporción de la población con acceso a un suministro seguro de agua potable. Este acceso puede ser de las siguientes formas: agua entubada, toma pública, pozos protegidos, corrientes protegidas o agua de lluvia.

\section{Justificación}

Sin duda, contar con acceso a agua segura es una de las necesidades más apremiantes, sobre todo si se considera un suministro adecuado en cantidad y calidad para garantizar aspectos de salud y supervivencia. La población que carece de dicho acceso es segregada irremediablemente, pues tal condición es suficiente para que en muchos casos les sean negados sus derechos. 


\section{Algoritmo}

AAS $=(1-($ OVPAP $/$ TOVP $)-\mathrm{NE}) \times 100$

Variables (con codificador de INEGI)

- OVPaP = Ocupantes en viviendas particulares que no disponen de agua entubada y usan agua en pipa.

- TOVP $=$ Total de ocupantes en viviendas particulares.

- $\mathrm{NE}=$ Ocupantes en viviendas particulares que no especificaron si disponían o no de agua potable.

\section{Cálculo}

AAS $=(1-(39,884 / 138,511-224) \times 100$

AAS $=96.44 \%$

\section{Acceso a instalaciones sanitarias adecuadas (AISA)}

\section{Definición}

Proporción de hogares con acceso a instalaciones sanitarias adecuadas.

\section{Justificación}

Es indispensable que una vivienda cuente con las instalaciones adecuadas para expulsar o separar del entorno doméstico los desechos humanos, lo cual reduce significativamente el riesgo de contraer enfermedades por el contacto con larvas, insectos, roedores o incluso animales domésticos y generar un ambiente propicio para vivir. El servicio sanitario también contribuye a incrementar el control de la contaminación de los cuerpos de agua y de los ecosistemas en general, pues induce las descargas en sitios específicos en los que posteriormente se aplicarán procesos de tratamiento para la eliminación paulatina de los residuos. 
Algoritmo

AISA $=($ OVSSECADRP + OVSSECADFS + OVSSEAMADRP + OVSSEAMADES $/$ TOVP $\mathrm{NE}) \times 100$

Variables

- OVSSECADRP $=$ Ocupantes en viviendas particulares con servicio sanitario exclusivo, conexión de agua y drenaje conectado a la red pública.

- OVSSECADFS = Ocupantes en viviendas particulares con servicio sanitario exclusivo, conexión de agua y drenaje conectado a fosa séptica.

- OVSSEAMADRP = Ocupantes en viviendas particulares con servicio sanitario exclusivo, admisión manual de agua y drenaje conectado a la red pública.

- ovsseamades: Ocupantes en viviendas particulares con servicio sanitario exclusivo, admisión manual de agua y drenaje conectado a fosa séptica

- $\operatorname{Tovp}=$ Total de ocupantes en viviendas particulares.

- $\mathrm{NE}=$ Ocupantes en viviendas particulares que no especificaron si disponían o no de sanitario exclusivo.

\section{Cálculo}

AISA $=(954,061+45,151+35,400+15,776 / 1,138,511-2,193) \times 100$

AISA $=92.43 \%$

\section{Conexiones domiciliarias (CD)}

\section{Definición}

Porcentaje de ocupantes en viviendas particulares que disponen de energía eléctrica, agua potable y drenaje conectados a la red pública. 


\section{Justificación}

Promover el acceso a los servicios básicos. El acceso a la infraestructura de servicios locales está relacionado con la calidad de vida y con los niveles de pobreza y riqueza. Así, la falta de conexión a servicios básicos hace que las comunidades que se encuentran viviendo en asentamientos informales sean particularmente vulnerables a enfermedades y epidemias.

\section{Algoritmo \\ $\mathrm{CD}=($ OVEAD $/$ TOVP $-\mathrm{NE}) \times 100$}

\section{Variables}

- $\quad$ TOVP $=$ Total de ocupantes en viviendas particulares.

- OVEAD = Ocupantes en viviendas particulares que disponen de energía eléctrica, agua potable y drenaje conectados a la red pública.

- $\mathrm{NE}=$ Ocupantes en viviendas particulares que no especificaron si disponían de agua entubada, energía eléctrica y drenaje.

\section{Cálculo}

$\mathrm{CD}=(985,583 / 1,138,511-1827) \times 100$

$\mathrm{CD}=86.7 \%$

\section{Precio del agua (PA)}

\section{Definición}

Precio medio del agua por metro cúbico de la tarifa para uso doméstico. Justificación

Administrar el suministro y demanda de agua de forma eficaz. 
Algoritmo

$\mathrm{PA}=($ TARIFAMAX + TARIFMIN $) / 2$

Variables

- TARIFAMIN $=$ Tarifa mínima del agua potable para uso doméstico por metro cúbico. Esta variable corresponde al rango de consumo doméstico de 0 a 20 metros cúbicos por mes (JMAS, 2005).

- TARIFAMAX = Tarifa máxima del agua potable para uso doméstico por metro cúbico. Esta variable corresponde al rango de consumo doméstico mayor a 151 metros cúbicos por mes (JMAS, 2005).

\section{Cálculo}

$\mathrm{PA}=3.10+13.97 / 2$

$\mathrm{PA}=8.5 \mathrm{pesos} /$ metro cúbico

Observaciones

El esquema actual de cobro del agua en un sistema de bloques establece una diferencia muy grande entre la tarifa mínima y la máxima. Creemos que un precio promedio más cercano a la realidad, o al menos a aquel que paga más del $90 \%$ de los usuarios, consistiría en tomar el promedio de los dos primeros bloques y reducir el precio a un costo aproximado de cinco pesos por metro cúbico.

\section{Consumo de agua (CA)}

Definición

Consumo de agua en litros por día y por persona para todos los usos domésticos (excluye el uso industrial, comercial y público).

Justificación

Administrar el suministro y demanda de agua en forma eficaz. 


\section{Algoritmo}

$\mathrm{CA}=\mathrm{VAFUDL} / \mathrm{TOVP}$

Variables

- $\operatorname{VAFUDM}^{3} /$ año = Volumen de agua anual facturado por concepto de uso doméstico en metros cúbicos $=115,633,582$.

- VAFUDL/día=Volumen de agua anual facturado por concepto de uso doméstico en litros por día.

- TOVP $=$ Total de ocupantes en viviendas particulares.

Estas variables se basan en JMAS e INEGI, XII Censo General de Población y Vivienda, 2000.

\section{Cálculo}

$\mathrm{CA}=316,804,334 / 1,138,511=278.26$ litros/hab./día.

Observaciones

La definición se alteró en función de los datos existentes en la JMAS, donde se mide el consumo de agua en función del monto facturado en pesos por categorías de usuarios.

\section{Aguas residuales tratadas (ART)}

Definición

Porcentaje de todas las aguas residuales que son sometidas a alguna forma de tratamiento.

Justificación

Reducir la contaminación en zonas urbanas y en los cuerpos receptores de los efluentes tratados. 
Algoritmo

$\mathrm{ART}=\mathrm{VTART} / \mathrm{VAR} \times 100$

Variables

- VTART $=$ Volumen total de aguas residuales tratado expresado en litros por segundo (lps). 81'645,655 m³/año.

- $\operatorname{VAR}=$ Volumen de aguas residuales expresado en litros por segundo (lps).

Estas variables se basan en cálculos de JMAS, JCAS y CNA.

\section{Cálculo}

$\mathrm{ART}=3,900 \mathrm{lps} / 3,400 \times 100$

$\mathrm{ART}=87 \%$

\section{Observaciones}

El dato del volumen de aguas residuales es una estimación de los volúmenes diarios y estimados en capacidades actuales.

\section{Resumen de indicadores}

Finalmente, todos los indicadores desarrollados y calculados son presentados en forma resumida en el cuadro 8 . Como puede observarse, no encontramos comparaciones en el tiempo ni con otras ciudades. La idea es que estos indicadores se puedan replicar en las principales ciudades con condiciones poblacionales similares a Ciudad Juárez y que cuenten además con organismos operadores de agua capaces de tener la información relevante que permita la construcción de más indicadores a fin de completar el esquema presentado por Bossel (1999), que incluye indicadores de Estado-Presión-Impacto-Respuesta con los cuales podría evaluarse la sustentabilidad en un contexto nacional. 
Cuadro 8.

Resumen de indicadores de uso sustentable del agua en Ciudad Juárez, Chihuahua (2000-2005)

\begin{tabular}{ll}
\hline Indicador & Estimación \\
\hline I. $\quad$ Acceso a agua segura (AAS) & $96.44 \%$ \\
II. Acceso a instalaciones sanitarias & \\
$\quad$ adecuadas (AISA) & $92.43 \%$ \\
III. Conexiones domiciliarias (CD) & $86.7 \%$ \\
IV. Precio del agua (PA) & $8.5 \mathrm{pesos} /$ metro cúbico \\
V. Consumo de agua (CA) & 278.26 litros/hab./día. \\
VI. Aguas residuales tratadas (ART) & $87 \%$
\end{tabular}

Fuente: Elaboración propia con datos del XII Censo General de Población y Vivienda, INEGI, 2000, y JMAS.

\section{Comentarios finales}

El alto crecimiento demográfico experimentado en Ciudad Juárez desde los años cincuenta a la fecha ha impreso el incremento directo en las demandas de agua potable para su crecimiento, así como incrementos constantes en la generación de aguas residuales. Los dos mayores retos en materia de agua para Ciudad Juárez son el abastecimiento seguro de agua potable en cantidad y calidad, así como el mantenimiento y aumento de la capacidad actual de tratamiento de sus aguas residuales. Lo anterior aunado a un escalamiento en el nivel de tratamiento; esto es, de primario a secundario y hasta terciario.

El sistema de agua potable de Ciudad Juárez manejado por la JMAS extrae el 100\% de su agua del acuífero de Bolsón del Hueco con una tasa de extracción que sobrepasa varias veces la tasa de recarga natural, por lo que se estima que el acuífero se encuentra bajo un régimen de sobreexplotación no sustentable y con consecuencias drásticas a corto plazo. Al momento no fue posible calcular un indicador de explotación/recarga dado que los únicos datos congruentes existentes tienen que ver con la extracción, faltando investigación en materia de recarga. Sin embargo, en cuanto a los indicadores desarrollados en este trabajo, Ciudad Juárez presenta información que manifiesta un 
aparente uso sustentable del agua y, en general, buenas condiciones en el manejo de la red de agua municipal.

Ciudad Juárez cuenta con una alta proporción de población con acceso a agua segura (AAS) y un suministro adecuado en términos de cantidad y calidad. Así, se estimó que un $96.44 \%$ de su población dispone de agua entubada o bien se le hace llegar en pipa. La población cuenta con un consumo de agua estimado en litros por persona por día de aproximadamente 278.26 litros. Esta cantidad rebasa los 250 litros por persona por día recomendados por organismos internacionales. Es importante destacar que el consumo per cápita de Ciudad Juárez equivale a un tercio del consumo en la ciudad de El Paso, Texas.

Respecto al indicador que mide el acceso a instalaciones sanitarias adecuadas (AISA), éste arroja una cobertura del 92.43\%. Se encontró que en esta ciudad las viviendas cuentan con instalaciones adecuadas para expulsar o separar del entorno doméstico los desechos humanos, contando con servicios sanitarios exclusivos, conexión de agua y drenaje conectado a la red pública.

El indicador que mide conexiones domiciliarias revela que $86.7 \%$ de la población cuenta con energía eléctrica, agua potable y drenaje conectados a la red municipal.

En materia de estimación del precio del agua, el indicador estimó que se cobra un precio medio de 8.5 pesos por metro cúbico. La JMAS maneja un esquema de cobro en bloques y en éste se establece una diferencia muy grande entre la tarifa mínima y la máxima, por esa razón la tarifa promedio estimada es muy alta. Sin embargo, se estima un precio promedio de cinco pesos el metro cúbico pagado por más del 90\% de los usuarios, esto es, los del consumo doméstico. En este renglón la junta de aguas cuenta con un potencial enorme para ingresos por concepto del cobro de agua. Asimismo, cuenta con un total de más de 350 mil usuarios conectados a la red municipal con coberturas de medición cercanas al 73\% y una capacidad de recaudación mayor al 70\%. También tiene un programa agresivo de instalación de medidores que permitirá a corto plazo la eliminación del cobro por cuota fija (en el actual sistema de bloques de consumo).

En materia de tratamiento de aguas residuales, se trabajó el indicador "Aguas residuales tratadas", que arrojó una proporción del 87\% 
de las aguas tratadas, no obstante que el constante crecimiento y su impacto en la mayor generación de aguas residuales rebasa la capacidad de tratamiento actual. Actualmente se tiene proyectada la construcción de más plantas de tratamiento, así como el escalamiento de tratamiento primario a tratamiento secundario en sus plantas principales.

En Ciudad Juárez se cuenta con buenos indicadores de uso sustentable del agua, sobre todo aquellos que tienen que ver con su manejo una vez que está dentro del sistema municipal de agua potable y saneamiento. Sin embargo, falta mucho por hacer en materia de indicadores de explotación sustentable y para asegurar suministros futuros a largo plazo. Se enfatiza la necesidad de contar con indicadores de extracción-recarga del sistema actual de bombeo. Falta además información para poder construir indicadores sobre calidad del agua potable. Actualmente, la JMAs de Ciudad Juárez no cuenta, o al menos no revela, la existencia de un programa de monitoreo constante sobre la calidad del agua servida.

\section{Bibliografía}

Bixby, K. (1999), "Water Conflicts in the Paso del Norte Border Region", Borderlines 57, disponible en http://www.us-mex.org/ borderlines/1999/b157

Bossel, Hartmut (1999), Indicators for Sustainable Development: Theory, Method, Applications, International Institute for Sustainable Development, Winnipeg, Canadá.

CGSNEGI, Carta de climas, 1:1000,000.

, Carta geológica, 1:1000,000.

CNA (2002), Registro mensual de temperatura media, INEGI, Anuario estadístico.

El Diario (2005), "Inicia JMAS instalación de 40,000 medidores", El Diario, 4 de julio de 2005.

El Paso Water Utilities (EPWU) (2006), "El Paso Water Supply", recuperable en línea: www.epwu.org/water/water_resourcces.html Fuentes Flores, César Mario y Luis E. Cervera Gómez (2004), “La gestión del suelo urbano en Ciudad Juárez, Chihuahua: la difícil 
transición de la gobernabilidad autoritaria a la gobernabilidad democrática", en Luis Antonio Payán y Socorro Tabuenca Córdoba (coords.), Gobernabilidad o ingobernabilidad en la región Paso del Norte, Universidad Estatal de Nuevo México, Universidad Autónoma de Ciudad Juárez, El Colef, Editorial EON, México. Fuentes Flores, César Mario (2001), "Los cambios en la estructura intraurbana de Ciudad Juárez, Chihuahua, de monocéntrica a multicéntrica", Frontera Norte, vol. 13, núm. 25, enero-junio.

Gleik Peter, H., Sandra Postel L. y Jason Morrison (1996), The Sustainable Use of Water in the Lower Colorado River Basin, The Pacific Institute for Studies in Development, Environment and Security, Oakland, California.

Heywood, C.E. y R.M. Yager (2003), Simulated Ground-Water Flow in the Hueco Bolson, and Alluvial-Basin Aquifer, El Paso, Texas: U.S., Geological Survey Water-Resources Investigations Report 024108.

Hibbs B., C. Eastoe, F. Philips y J. Hogan (2002), “Groundwater and Surface Water Salinization in the El Paso/Juarez Region. Thrust Area 2 Overview. SAHARA. Disponible en línea: http://www. sahra.arizona.edu/research/TA2/2_9.html

IBWC (1998), "Transboundary Aquifers and Binational Ground-Water Data Base. City of El Paso/Ciudad Juárez Area", disponible en línea: http:/ / www.ibwc.com

INEGI (2000), XII Censo General de Población y Vivienda, IV-Chihuahua, Aguascalientes, México.

(2002), Anuario estadístico del estado de Chihuahua, Aguascalientes, México. (2002), Anuario estadístico, Chihuahua.

JMAS (1997), "Proyecto de las Plantas de Tratamiento de Aguas Residuales Norte y Sur y Obras Complementarias de Alcantarillado de Cd. Juárez, Chihuahua", Formato Etapa II de COCEF, disponible en línea: www.cocef.com (2002), "Condición de agua potable y saneamiento en Juárez", documento inédito. (2005), Plan de trabajo 2005, Gobierno del Estado de Chihuahua. 
JMAS (2000), "Alternativas presentadas en el Plan Maestro para el Mejoramiento de los Servicios de Agua Potable, Alcantarillado y Saneamiento, Ciudad Juárez".

Loucks, P. Daniel y John Gladwell S. (1999), Sustainability Criteria for Water Resource Systems, International Hydrology Programme, Cambridge University Press.

OECD (1998), Toward Sustainable Development: Environmental Indicators. Francia, OECD.

Rincon, C. et al. (2005), "Promoción de asociaciones de colaboración participativa para la conservación del recurso agua en la región Paso del Norte", Paso del Norte Water Task Force (PNWTF), documento en revisión para su publicación en formato electrónico.

Plan Municipal de Desarrollo 2002-2004. Aprobado por el H. Ayuntamiento en sesión extraordinaria el 19 de noviembre del 2002.

Fecha de recepción: enero de 2006

Reenviado en: junio de 2006

Fecha de aprobación: enero de 2007 